\title{
EDITORIAL
}

\section{Collateral damage: the conundrum of drug safety}

\author{
E. A. M. Gale
}

Published online: 18 August 2009

(C) Springer-Verlag 2009

Keywords Diabetes · Diabetes therapies · Drug safety . Exenatide $\cdot$ Pharmacovigilance

\section{A ripple from the storm}

Drugs for health generate wealth, and wealth drives the diabetes roadshow. Without it, the subsidised seasonal migrations to our big meetings would grind to a halt, and the KOLs (Key Opinion Leaders, for the uninitiated) would all be driving a different brand of car. Given that the USA absorbs one in every two drug dollars expended on this planet, it is no surprise that the American Diabetes Association (ADA) meeting provides the premier catwalk upon which the latest drug models strut their stuff. The meeting is followed by a series of debriefing sessions in which big names in diabetes are courted by investors eager to learn the latest gossip.

On 11 June 2009, Dr Ralph DeFronzo imparted wisdom to a select group of investors, somewhat in the style of Moses descending from the mountain. His pronouncements concluded with a cryptic comment that would leave the financial journalists scrambling for their insider contacts. 'The glargine world is about to be shipped up in a big way, a huge way', he remarked. 'This will be an earthquake larger than any earthquake you've ever seen in the world when it hits.' [1]. Coincidentally-I don't believe he was referring to us-Diabetologia would

E. A. M. Gale ( $\square)$

Diabetes and Metabolism, Medical School Unit,

Southmead Hospital,

Bristol BS10 5NB, UK

e-mail: Edwin.Gale@bristol.ac.uk publish four studies on the safety of insulin glargine only 2 weeks later [2]. Shares in sanofi-aventis dropped by $€ 7.7$ billion ( $\$ 10.8$ billion), $12.3 \%$ of their total value, in the 2 days preceding our announcement - which came earlier than planned because of a leak to a journalist. This brief interval of free fall was not, as should be noted, in response to any new information: simply a combination of DeFronzo's remark and rumours of our forthcoming publication (Fig. 1).

Diabetologia sought to put the studies it had received in the public domain with the minimum of fuss, and in this we were helped by the untimely demise of Michael Jackson on the day we broke the news. It also helped that we hold a relatively modest position in the pecking order of journals. If Nissen and Wolski had published their notorious rosiglitazone meta-analysis in Diabetologia rather than the New England Journal of Medicine [3], I suspect that we might be using a lot more of it today. Our reports were, for the most part, reported and received with all the appropriate reservations, and will, as we hope, lead to the full-scale investigation needed to resolve this issue.

\section{Two steps forward, one step back}

In 1990 we had only three classes of drugs for diabetes: insulin (animal or human), sulfonylureas and metformin. Nine classes are now available [4]. The newer agents have expanded the range of clinical choice, with individual benefit to many patients, but they have also greatly increased the cost of treatment [5]. The recent ADA/EASD consensus statement concluded that newer agents do not lower glucose more effectively than the three traditional therapies [6], and that they have no demonstrated impact 


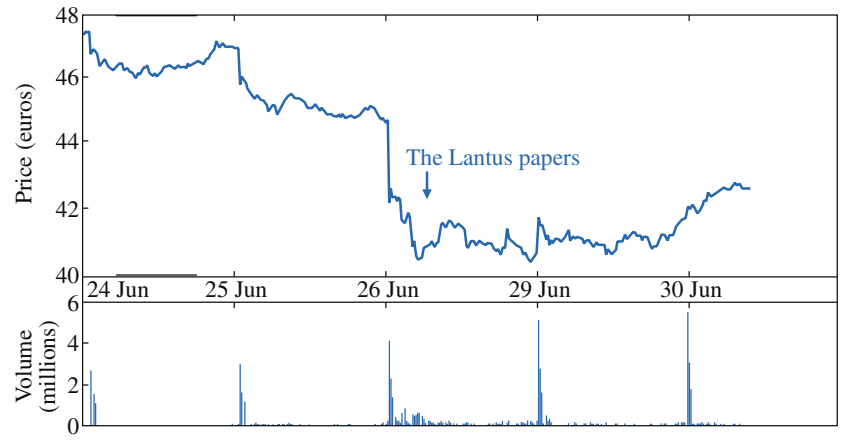

Fig. 1 Shares in sanofi-aventis dropped by $€ 7.7$ billion ( $\$ 10.8$ billion), $12.3 \%$ of their total value, on the rumour of a safety publication. They have since returned to their previous value. From http://uk.finance. yahoo.com, copyright 2009 Yahoo!

upon the prognosis of type 2 diabetes. We are therefore in a situation of escalating costs for diminishing returns (Fig. 2).

There is an undeniable tendency for the clinical benefits of each new agent to be overvalued at the point of entry to the market. Here are some of the reasons: (1) many new therapies arise from important advances in scientific understanding, and it is natural to anticipate commensurate clinical benefit; (2) the drug development process highlights efficacy, whereas it takes longer for the unwanted consequences of new therapies to emerge; (3) head-to-head comparisons with existing therapies are typically lacking [6]; (4) the drug companies only have a short window of opportunity in which to recoup investment and profit from their licence, and therefore seek to saturate the market at the earliest possible opportunity; (5) our patients are accustomed to the fact that each new generation of cars, computers and mobile phones is better than the last, and tend to assume the same about new drugs; (6) we underestimate the sheer power of suggestion upon our own behaviour and that of our patients [7]; recruitment into a clinical trial produced a greater fall in $\mathrm{HbA}_{1 \mathrm{c}}$ in one batch of clinical trials than the intervention itself [8]. No surprise, therefore, that new drugs enter the clinical arena against a background of inflated expectation coupled with unrealistic assumptions as to safety.

The eternal dilemma of the clinician is to weigh the potential benefits of each new therapy against its disadvantages and cost, and to make the balance as favourable as possible for each individual who comes seeking advice. Since none of the newer agents for diabetes offers clear advantages over the old in terms of glucose-lowering potential, and outcome studies are lacking, the prescriber's choice will be determined by patient preference, qualitative differences (e.g. risk of hypoglycaemia), off-target effects (whether wanted or unwanted), cost, and (above all) by perceived safety.

Safety considerations impact at three distinct levels: financial, regulatory and clinical. From the financial point of view, each new therapeutic agent represents a gamble, with billions of dollars riding upon the outcome, and serious adverse events an ever-present threat. From the regulatory point of view, each new drug is a leap in the dark, with possible harm to large numbers of people. For the clinician, each new drug prescribed is a one-off exercise in clinical probability based upon incomplete information. Most analyses of drug safety focus on the role of the regulatory authorities, but this article will focus on the relatively neglected roles of the money market and of the clinician as proxy consumer.

\section{The maiden voyage of the Titanic}

Captain E. J. Smith of the Titanic once made the immortal comment that his 40 years at sea had been entirely uneventful. 'I never saw a wreck', he said, 'and never have been wrecked nor was I ever in any predicament that threatened to end in disaster of any sort.' [9]. The Titanic was a supposedly unsinkable ship that set out to break the transatlantic speed record on its maiden voyage, which is why its captain ordered full steam ahead despite a warning about icebergs. The CEO of a pharmaceutical company is under similar pressure, for his or her professional duty is to deliver increasing value to shareholders. As for icebergs: What icebergs?

Icebergs, real or imagined, lie in the path of all new drugs. Within the field of diabetes, troglitazone and rimonabant have been withdrawn, and rosiglitazone, exenatide and insulin glargine have taken hits, fairly or unfairly, from safety concerns. The financial consequences are almost incalculable - shares in sanofi-aventis, as we have seen, lost $\$ 10.8$ billion in value on the basis of mere rumour. Shares in GlaxoSmithKline took a nosedive following the Nissen and Wolski meta-analysis of rosiglitazone [3] (Fig. 3), followed by a smaller dip in response to

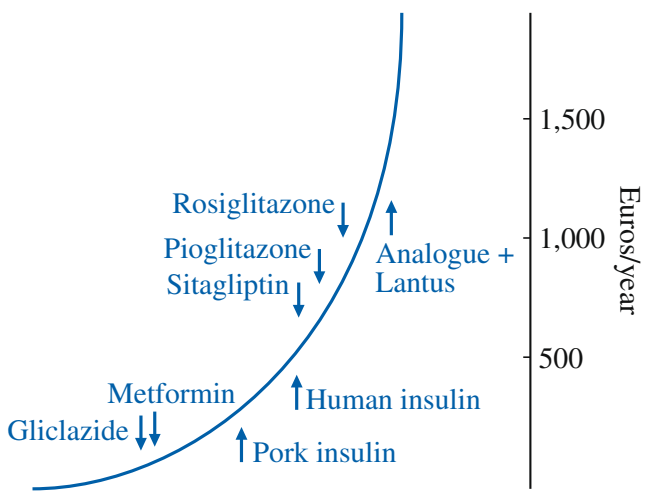

Fig. 2 Newer treatments cost more: estimated annual cost of diabetes therapies in the UK, 2007 (costs obtained from the British National Formulary) 


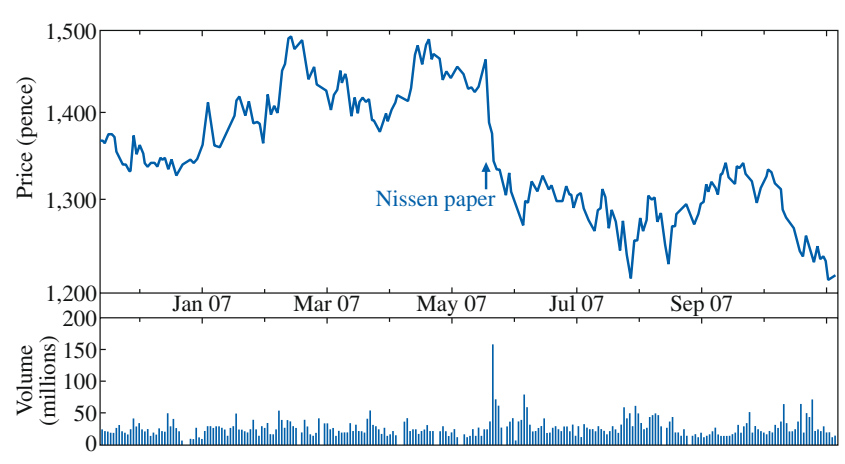

Fig. 3 The impact of the Nissen and Wolski paper [3] on the share price of GlaxoSmithKline. From http://us.finance.yahoo.com, copyright 2007 Yahoo!

the ADA/EASD consensus statement [6] in January of this year (Fig. 4). Sales of rosiglitazone fell from $\$ 1.6$ billion in 2006 to $\$ 805$ million in 2008. Amylin, a much smaller company, survived a near-death experience in 1998 when staff numbers fell from 300 to 37 , and the survivors were obliged to invest their own savings to keep the company afloat $[10,11]$. Against all the odds, it recovered and was heading towards $\$ 1$ billion in sales in 2009 before it encountered the pancreatitis iceberg. The company was obliged to lay off 340 staff in November 2008 [12]. It is easy to see why a relatively small enterprise such as Amylin might suffer, but why are the big companies so vulnerable?

\section{Vulnerable giants}

The answer is that each company derives its income stream from a handful of blockbuster drugs, and is reliant upon investor confidence. 'Investor confidence' is an oxymoron, for investors are the most neurotic people imaginable. The economist John Kenneth Galbraith accurately prefigured the future of the pharmaceutical industry in a book titled American Capitalism, which he published in 1952 [13]. He pointed out that classic economic theory assumed open competition, which would guarantee maximum efficiency as companies competed to generate the best or cheapest product. This was not how things worked in the real world, however, as Galbraith noted, for market sectors rapidly come to be dominated by producers who control the price of their own product. This happens because larger companies enjoy economies of scale, which allow them to grow faster and to invest more in research. They then consume weaker rivals until only a few enormous corporations remain. Competition ceases once these control the market, for there is no point in trying to undercut your rival when the price of your products can be fixed at a mutually advantageous rate. To take one example, three big companies dominate the insulin supply. The price of insulin varies widely from one country to the next, but you will find that competing products are pegged at much the same level in the same market.

A pharmaceutical oligopoly might seem to be a recipe for stability, but this is not the case. The novice investor judges pharmaceutical companies by their return on investment, but the real money comes from buying and selling shares. Stock market investors typically invest in undervalued companies or companies with strong growth prospects. This produces significant volatility, since share prices are based upon expectation of future share prices, and expectations can change within seconds based on new information, or concerns about new information. Investors know that each company's fortunes depend upon a few branded drugs, each with a limited patent life. Hence the endless lawsuits designed to exclude real competition from the manufacturers of generic or imported products, and the equally endless quest to 'evergreen' top-selling drugs by tweaking the formulation or indication in order to renew their licence. Investors take an avid interest in the drug discovery pipeline, and flock around a promising development like vultures on a fence, always ready to take flight at the first hint of trouble. This instability creates a rollercoaster ride on the stock market, and generates big profits for those who know when to sell the market short.

Investment advisors go to considerable lengths to investigate possible safety threats, as I shall shortly demonstrate, and are generally better informed than any practising physician. A pharmaceutical company, on the other hand, will be reluctant to concede the possibility of harm, for fear that this might send an alarm signal to investors. The company may then find itself vulnerable if a serious unwanted effect does come to light, and will suffer disproportionate damage. The regulatory authorities, meanwhile, move at a deliberative pace and are well aware of the commercial sensitivity of the statements they issue. When these watchdogs begin to bark, you may well find that the intruder is already inside your house.

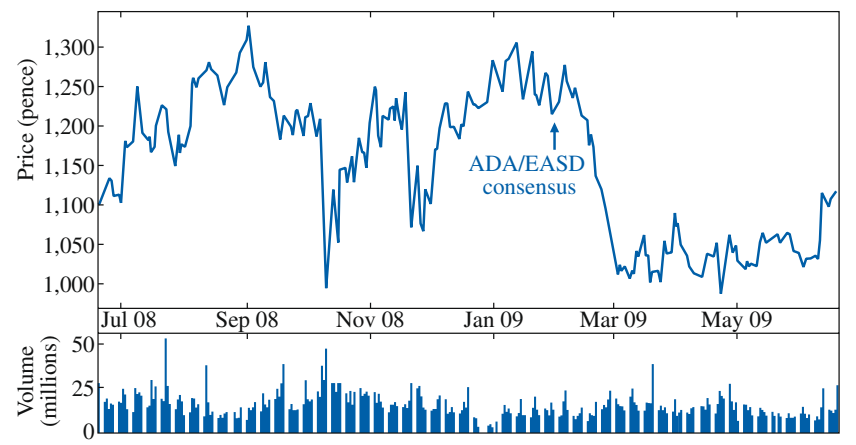

Fig. 4 The impact of the ADA/EASD consensus statement [6] on the share price of GlaxoSmithKline. From http://us.finance.yahoo.com, copyright 2009 Yahoo! 
Marketing sensitivities distort our whole approach to drug safety. A rational system would focus upon the need to protect individual patients from possible harm, rather than upon the need to allow investors to sleep soundly at night. A rational system would acknowledge that all potent medications have undesirable effects, and would devote its energy to identifying and investigating these at an early stage. It would alert patients and physicians to possible danger at the earliest possible moment, rather than afterwards.

\section{Catch-22}

Each new drug is an experiment [14]. Phase 2 studies, conducted in a few hundred patients, demonstrate efficacy. Phase 3 studies, typically performed in 2,000 to 5,000 patients, are designed to identify major potential safety concerns. They define a safety threshold that the drug must clear before it enters the market, but they can provide no guarantee of safety. Trial participants are typically healthier than real patients, and rare adverse events $(<1 / 1,000$ patient-years $)$ will not be detected reliably, especially if the adverse effect is consistent with the natural history of the condition being treated (e.g. suicide in depression, or heart disease in diabetes). In the event, clinically important post-marketing safety concerns have emerged for $50 \%$ of approved drugs [15]. This, somewhat unfairly, is seen as a failure of regulation rather than a success for pharmacovigilance.

Troglitazone and rimonabant : rosiglitazone, exenatide, insulin glargine. It is a worrying catalogue of safety concerns. Worrying because we may have exposed our patients to avoidable danger, yet - in some cases at least - we are left to wonder what that danger actually was. Worrying because we are force-fed with the stated benefits of new agents, while potential or emerging hazards are understated or even concealed. Worrying because safety is defined by a legalistic and adversarial dialogue between the companies and the regulatory authorities, with doctors and patients relegated to the sidelines. Worrying because commercial secrecy may trump the public interest when it comes to information affecting our health [16]. Worrying because popular and valued therapies for diabetes are vulnerable to fears that may have no substance, and may (like rosiglitazone) be put to the sword on grounds of mere suspicion. Worrying because risk can be evaluated, but safety can never be proved.

The regulatory authorities might do well to adopt 'Catch$22^{\prime}$ 'as their motto, for they are at one and the same time accused of delaying access to valuable new drugs by insisting upon unnecessary screening procedures, and of exposing the public to unnecessary risk because these same screening procedures are inadequate. As this might suggest, they are in fact not doing too badly; and proposed new legislation could greatly reinforce the developing role of the European Medicines Evaluation Agency (EMEA) [17]. The one issue on which all concerned can agree is that postapproval safety monitoring of all new drugs could be much better than it is, a point of view most clearly and exhaustively set out in a lengthy report and set of recommendations from the US Institute of Medicine [18]. Various remedies or improvements have been proposed, piloted or implemented, but few would deny that the current system leaves something to be desired. Let us pause to consider it.

The pharmaceutical companies carry formal responsibility for monitoring the safety of their drugs, and for informing physicians of possible risks, despite the obvious conflict of interest involved in asking them to act as consumer watchdogs for their own products. A company might well be disinclined to expose itself to loss of sales and litigation by digging too deeply for evidence of possible harm. The regulatory authorities, in dialogue with the companies, represent the second line of defence. Specific analyses may be requested; for example, the post-marketing comparison of diabetic retinopathy in those on insulin glargine and human insulin [19], but the system otherwise relies on spontaneous reports. Europe has the handicap that each member state currently operates its own drug vigilance system which feeds into a central database; the USA has an Adverse Event Reporting System (AERS), fed by reports submitted electronically by company representatives, healthcare workers or members of the public. The resulting blizzard of information must be carefully sorted, checked and verified before further analysis. Ascertainment of adverse events is low in all such systems, at around $1 \%$ of the true frequency [20], although reports are more likely to be submitted for new drugs or recently publicised complications. A further line of defence is provided by the medical journals. Case reports once played an important role in drug safety, and (as we will see) a single case report played an important role with respect to exenatide, but the clinical case report has largely fallen out of fashion. Independent investigation of a safety issue, as undertaken recently by this journal, is excessively rare. Consumer watchdogs such as Public Citizen play an important gad-fly role, but other so-called consumer action groups are funded by the pharmaceutical manufacturers.

There are, in other words, many anomalies and perverse incentives within the drug safety system. Companies are legally responsible for the safety of their own product, but they are also legally obliged to maximise profit for their investors. Catch-22. The regulatory authorities need to provide early warning of possible adverse effects, yet cannot act on incomplete information. Catch-22. And so it 
goes. Let us, therefore, look at one example of the system in action.

\section{Drug safety? Ask an investor}

The Food and Drug Administration (FDA) approved exenatide on 29 April 2005. A single case report appeared in February 2006 [21], and a second followed in an Indian journal in 2008 [22]. The company added a note about pancreatitis to the post-marketing section of the label on 12 October 2006, and the FDA issued its first alert, based on 30 post-marketing reports of acute pancreatitis, in October 2007 [23]; this was subsequently updated with news of six further cases of haemorrhagic or necrotising pancreatitis (two of them fatal) in August 2008 [24]. A letter from the FDA, published in May 2008, represented the first accessible public report [25], and the FDA house journal described a further case later in the year [26]. A safety review published in 2007 [27] mentions the single published case [21], and an update published in the following year describes the FDA findings, whilst noting that pancreatitis was inadequately documented in many of the safety reports [28]. Clinical trials data supplied to me by the company indicate that the programme has identified eight cases of pancreatitis on exenatide and two on placebo or comparator, although the base rate is said to be similar when adjusted for the numbers in either group. Two more recent surveys, each with company funding or other links, suggest that acute pancreatitis may be no more common in those on exenatide than it is in the rest of the type 2 diabetic population $[29,30]$. Does the problem not even exist? Meanwhile, a recent safety update reports a total of 396 cases worldwide by September 2008, for an estimated exposure of 800,000 patient-years, with nine reports of haemorrhagic pancreatitis [31]. So where does this leave us? How much of a threat is there? This is the state of public information 4 years after the drug reached the market.

So much for the information available to doctors and patients. Now let us examine the service provided to investors by investment analysts working for the investment bankers. The first case report in 2006 prompted several finance houses to investigate, and the Freedom of Information Act allowed them the means to do so. Exactly 1 year before the FDA alert, on 2 October 2006, Bear Stearns reported that 'many market participants have evaluated the FDA's AERS database using various screening and data-mining techniques. The process threw out a large number of reports of Byetta patients suffering from pancreatitis'. The safety notifications were then subjected to a labour-intensive manual search that identified 26 likely cases of pancreatitis. The analysis that followed was of roughly the same length as this Editorial, and claimed that the spontaneous reporting rate for pancreatitis with exenatide was $68 / 100,000$ patient years, well above the background rate of $17 / 100,000$. Two other diabetes agents were used as comparators, and pancreatitis was reported 37 times more frequently with exenatide than with insulin glargine. The authors are at pains to point out that such comparisons must be viewed with considerable caution [32].

These adverse event reports are on the desk before me as I write. Their limitations are obvious, for the evidence supplied is patchy, and accurate follow-up information was often lacking. The same patient may have been reported by different people, and many were taking multiple drugs for multiple afflictions; other drugs also have possible links to pancreatitis. Some physicians actually refused to supply further details when other health professionals had notified their cases. All of which testifies to the difficulties encountered by those who have the monumental task of sifting through this information. Nonetheless, my own analysis makes it reasonably clear that at least 18 spontaneous reports of probable acute pancreatitis in patients on exenatide had been received within a year of launch, excluding duplicates and three other patients with a previous history of pancreatic problems, and that the episode presented within 30 days of starting or increasing the dose of exenatide in 11 of these cases.

This is not the place for a detailed discussion of exenatide and pancreatitis, which merits an Editorial in its own right. It is, however, clear that, real or otherwise, the risk of pancreatitis is not well documented. In the first place, the original FDA reports show that only 21 of 30 cases were admitted to hospital, only 17 had an amylase test performed (which was sometimes normal), and that 27 had other risk factors for pancreatitis. The next point to make is that we are not well informed as to the base rate of acute pancreatitis in the diabetic population; exactly the same issue arose when troglitazone was seen to cause acute liver failure.

I suspect that the focus on acute pancreatitis resulted from the myth that it is produced by the bite of the Gila monster, and that this focus has obscured a more fundamental safety concern. My own review of the AERS suggests that introduction or dosage adjustment of exenatide is (rarely) associated with a syndrome of acute circulatory collapse, vomiting and abdominal pain. In some cases this may have led to acute renal failure (seven cases reported in the UK, as against nine cases of pancreatitis) [31], coronary insufficiency or death. Such episodes appear more likely when exenatide is inappropriately prescribed for patients with multiple health problems. This is the sort of information that practising physicians really need to have, yet the problem has not even been framed in this way, let alone investigated. Meanwhile, we can observe that 
investors saw a detailed analysis of health concerns related to exenatide 12 months before the FDA notified physicians of the same possible hazard. There could be no clearer confirmation of the fact that financial risk triggers a much higher level of investigation and concern than a risk to patient safety.

There is nothing at all new about this delay in the transmission of safety information. The troglitazone debacle was remarkable, among other things, for the fact that the safety data in 1997 were sufficiently compelling for the CEO of Glaxo (as it then was) to pull the agent from the UK market within 1 month of its launch, yet it remained in use in the USA and Japan for two further years, during which time the initial safety concerns were amply confirmed [33]. Physicians were never told the full story about troglitazone, and there is more to learn about exenatide.

\section{The pen of the prescriber}

Visitors to the British Museum will encounter a display showing the 14,000 prescription tablets that a typical member of our society will consume in the course of a lifetime. One hand-sewn strip shows the tablets consumed by a composite man, who died of a stroke at the age of 75 . The other shows the tablets taken by an 82-year-old woman with diabetes. Rows of empty pockets remain for the pills she has yet to consume [34]. How many of these tablets were truly useful or necessary? Many years ago a radical guru called Ivan Illich wrote a book entitled Limits to Medicine, which argued (among other things) that old age and death were not medical conditions, and that indiscriminate use of potent medications was creating more damage than benefit [35]. Few physicians will follow the latter part of the argument to its final conclusion, but many of us suspect that he had a point. Illich, incidentally, fought a long drawn-out battle against a painful and disfiguring cancer before his death at the age of 76 , refusing the painkillers and sedatives offered by his doctors because they would interfere with his work [36].

Physicians are proxy consumers. We control the market for products that we neither pay for nor consume, and whose unwanted consequences are experienced by other people. The one inalienable certainty is that the doctor who writes the prescription is responsible for the consequences, whether anticipated or not. The 'golden rule' in other walks of life is to treat others as we would wish to be treated, and I cannot help noticing that most doctors of my acquaintance are extremely reluctant to take any form of medication. When we prescribe for patients, we think of possible benefit; when we prescribe for those near and dear to us, we recall that minor side effects can be defined as those which cause no discomfort to the prescriber. The only safe pill is the one you never prescribed.
Pills are taken for many reasons. Some relieve symptoms, others protect against clear and imminent danger, such as rejection of a transplanted kidney. There are pills for remoter possibilities, such as bony fractures, peptic ulcers or strokes, and pills for longevity. Longevity pills represent a ruthless determination to impose the metrics of a 25-year-old upon those who have already beaten the odds by surviving for 80 years or more. Possibility pills and longevity pills have their place in the scheme of things, but their use requires common sense and an understanding of simple probability, two characteristics that are in short supply in our profession.

There are, let me suggest, seven deadly sins of drug prescription. These are: (1) to use pharmaceuticals to treat a non-pharmaceutical problem; (2) to assume that new drugs are better; (3) to repeat prescriptions that serve no rational purpose; (4) to use one drug to counter the side effects produced by another; (5) to overestimate the benefits of your intervention; (6) to pursue the mirage of longevity beyond the realms of common sense; and (7) to reduce the quality of the life you are trying to improve. Not one of us is innocent of these sins, and drug safety would be much less of an issue if we were.

\section{Countervailing power}

John Kenneth Galbraith pointed out that a producer rises to wealth by gaining control of the supply side of the market. Retailers respond by consolidating to gain effective control of demand. One corporation gains control of the food supply, another creates a huge supermarket chain to force the price down. An oligopoly of suppliers is soon confronted by an oligopoly of distributers. Galbraith referred to this development as 'countervailing power' [13]. The same process operates within the health market, where large-scale suppliers of medication encounter largescale healthcare providers. Within the UK, for example, the National Health Service has a near-monopoly of healthcare provision, and can negotiate relatively competitive rates for the drugs it buys. Useful though it is, this nanny function cuts both ways. On the one hand, the National Institute for Clinical Excellence (NICE) can attempt (with only partial success) to impose cost-effective prescribing; on the other, primary care physicians are offered financial incentives to prescribe the possibility and longevity pills that diminish the quality of life of our elderly citizens, and put so many into hospital.

We are all consumers of health care, and our interests are best served when a reasonable balance exists between power and countervailing power; i.e. between the drug manufacturers and the regulators. But what about the physicians? And who speaks for an overmedicated public? 
The dialogue between companies and regulators takes the form of an elaborate ritual performed between mirror-image bureaucracies, and it is hard to avoid the conclusion that the rest of us have been relegated to the sidelines. In the last analysis, it all boils down to a question of who owns the data, and here we encounter a clash of cultures. The culture of large companies is driven by competition and commercial secrecy. The culture of the regulators is driven by a need to control the flow of information reaching the decision makers and the public, and this has both positive and negative aspects; positive when it avoids unwarranted alarms, negative when it delays the release of important information.

\section{Can we do better?}

Cultural problems have cultural solutions, and my sense is that the wider culture of our society is driving this issue forward in a very positive way. Our wider culture will return a clear answer to the question of who owns the data: where health and well-being are concerned, we all do. This question having been answered, the next challenge is to find better ways to acquire, evaluate and share data. These are technical questions, and solutions are beginning to emerge. One high priority is to bridge the approval watershed. We do not need larger or longer preregistration studies, but we do need a better system of ongoing vigilance once an agent reaches the public, possibly mediated by provisional registration, and supported by mandatory independent pharmacovigilance. We also need greater transparency, a buzz-word that is often on the lips of those who mean nothing more than decreased opacity. The websites of the FDA and EMEA have elevated 'concealing by revealing' into an art form. Finally, the regulators need to be more accessible. There was, for example, no formal pathway by which this journal could engage in dialogue with the regulators in the course of our investigations of insulin glargine. Despite goodwill on both sides, we either surrendered the information, or we did not. We did, and one national agency promptly leaked it to the press, forcing us to publish early. In summary, drug safety is everyone's problem, and physicians and the public need to be in the loop. The technical capacity for a better system already exists, but we need a culture shift in order to use it more effectively.

Further cultural shifts are necessary, and may already be under way. A mature and informed drug-consuming public should be aware that no drug is both safe and effective. Prescribers should neither be shielded from the knowledge of possible ill-effects, nor whipped into hysteria because these are under consideration. Patients need to be involved in such discussions. Woloshin and colleagues have shown that it is possible to construct simple tables of benefit, risk and unwanted effects of commonly used medications for patient use [37]. This could easily be done for diabetes, and would add new meaning to the concept of informed consent. Physicians involved in the care of diabetes, meanwhile, should be more ready to accept that most of the new therapies for diabetes have comparable and relatively modest glucose-lowering properties [6]. Choice should therefore be based upon patient preference outcome measures (which we lack) and a realistic appreciation of known and possible unwanted effects, about which we need to know more. Patient safety has been the core business of doctors since the days of Hippocrates, and we should never be persuaded otherwise.

\section{Envoi}

The biomedical revolution has unfolded with disconcerting rapidity, but some things have not changed. In the 1970s I visited a distinguished Irish physician who had retired to a grand but decrepit family mansion in the hills near Cork. He warned me to avoid a broken floorboard as I stepped into the hall; it broke when his bride stepped into the house on their wedding day 50 years earlier. He still planned to get it fixed. In 1909 or thereabouts he visited Oxford as a medical student, and paid a courtesy call on William Osler, then Professor of Medicine. Characteristically, Osler took him straight round to see an interesting patient. I asked what Osler had said, and he cast his mind back to that ghostly conversation. 'Osler told me this', he replied: 'Never be the first, or the last, to use a new drug.'

Duality of interest The author declares that there is no duality of interest associated with this manuscript.

\section{References}

1. Torsoli A (2009) Sanofi shares slump; analysts cut ratings in Lantus. Available from http://www.bloomberg.com/apps/news? pid $=20601085 \&$ sid $=$ aEiby 7 t0.yXc, accessed 29 July 2009. I have verified the quote from a transcript of the call

2. Smith U, Gale EAM (2009) Does diabetes therapy influence the risk of cancer? Diabetologia 52:1699-1708

3. Nissen SE, Wolski K (2007) Effect of rosiglitazone on the risk of myocardial infarction. N Engl J Med 356:2457-2471

4. Nathan DM (2007) Finding new treatments for diabetes-how many, how fast, how good? N Engl J Med 356:437-440

5. Hauber A, Gale EAM (2006) The market in diabetes. Diabetologia 49:247-252

6. Nathan DM, Buse JB, Davidson MB et al (2009) Medical management of hyperglycaemia in type 2 diabetes: a consensus algorithm for the initiation and adjustment of therapy. A 
consensus statement from the American Diabetes Association and the European Association for the Study of Diabetes. Diabetologia 52:17-30

7. Gale EAM (2004) The Hawthorne Studies: a fable for our times? QJM 97:439-449

8. Gale EAM, Beattie SD, Jinghui Hu, Koivisto V, Tan MH (2007) Recruitment to a clinical trial improves glycemic control in patients with diabetes. Diabetes Care 30:2989-2992

9. http://www.famousquotessite.com/famous-quotes-3210-e-j-smith, accessed 29 July 2009

10. Gale EAM, Clark A (2000) A drug on the market? Lancet 355:61-63

11. Weintraub A (2006) Bucking the odds at Amylin. Business week. Available from http://www.businessweek.com/magazine/content/ 06_02/b3966092.htm, accessed 29 July 2009

12. Flagging Byetta sales force job cuts at Amylin. 12 November 2008. Available from http://www.newsinferno.com/archives/4183, accessed 29 July 2009

13. Galbraith JK (1993) American capitalism. The concept of countervailing power. Transaction Publishers, Edison. Originally published in 1952 by Houghton Mifflin, Boston

14. Stricker BH, Psaty BM (2004) Detection, verification and quantification of adverse drug reactions. BMJ 329:44-47

15. Moore TM, Psaty BM, Furberg CD (1998) Time to act on drug safety. JAMA 279:1571-1573

16. McGoey L, Jackson E (2008) Seroxat and the suppression of clinical trial data: regulatory failure and the uses of legal ambiguity. J Med Ethics 35:107-112

17. Commission of the European Communities (2008) Proposal for a directive of the European Parliament and of the Council amending, as regards pharmacovigilance, Directive 2001/83/EC on the Community Code relating to medicinal products for medical use. Available from http://eur-lex.europa.eu/LexUriServ/LexUriServ.do? uri=COM:2008:0665:FIN:EN:PDF, accessed 29 July 2009

18. Committee on the Assessment of the US Drug Safety System (2007) The future of drug safety. Institute of Medicine of the National Academies, National Academies Press, Washington

19. Rosenstock J, Fonseca V, McGill JB et al (2009) Similar progression of diabetic retinopathy with insulin glargine and neutral protamine Hagedorn (NPH) insulin in patients with type 2 diabetes: a long-term, randomised, open-label study. Diabetologia. doi:10.1007/s00125-009-1415-7

20. Gottlieb S (2005) Opening Pandora's pillbox: using modern information tools to improve drug safety. Health Aff (Millwood) 24:938-948

21. Denker PS, Dimarco PE (2006) Exenatide (exendin-4)-induced pancreatitis. Diabetes Care 29:471
22. Tripathy NR, Basha S, Jain R, Shetty S, Ramachandran A (2008) Exenatide and acute pancreatitis. J Assoc Phys India 56:987-988

23. US Food and Drug Administration (2007) Exenatide (marketed as Byetta). FDA Alert (10/2007). Available from http://www.fda. gov/Drugs/DrugSafety/PostmarketDrugSafetyInformationfor PatientsandProviders/Drug SafetyInformationforHeathcare Professionals/ucm084305.htm, accessed 29 July 2009

24. US Food and Drug Administration (2008) Exenatide (marketed as Byetta) - Healthcare Professional Sheet text version (8/ 2008). Available from http://www.fda.gov/Drugs/Drug Safety/ PostmarketDrugSafetyInformationforPatientsandProviders/ ucm124713.htm, accessed 29 July 2009

25. Ahmed SR, Swann J (2008) Exenatide and rare adverse events. N Engl J Med 358:1969-1970

26. Exenatide (marketed as Byetta) Acute pancreatitis. FDA drug safety newsletter, Winter (2008). 12-14

27. Stephens JW, Bain SC (2007) Safety and adverse effects associated with GLP-1 analogues. Expert Opin Drug Saf 6:417-422

28. Bain SC, Stephens JW (2008) Exenatide and pancreatitis: an update. Expert Opin Drug Saf 7:643-644

29. Noel RA, Braun DK, Patterson RE, Bloomgren G (2009) Increased risk of acute pancreatitis and biliary disease observed in patients with type 2 diabetes: a retrospective cohort study. Diabetes Care 32:834-838

30. Dore DD, Seeger JD, Chan KA (2009) Use of a claims-based active drug surveillance system to assess the risk of acute pancreatitis with exenatide or sitagliptin compared to metformin or glyburide. Curr Med Res Opin 25:1019-1027

31. Pocock N (2009) MHRA drug safety update: Risk of severe pancreatitis and renal failure with exenatide $\left(\right.$ Byetta $\left.{ }^{\mathbb{R}}\right)$. Drug safety update, Volume 2, Issue 8

32. Hauber A, Wan K (2006) Type II diabetes: our thoughts on the GLP-1 opportunity. Bear Stearns, equity research report, 2 October 2006. Available from www.diabetologia-journal.org/ files/BearStearnsReport.pdf, accessed 11 August 2009

33. Gale EAM (2006) Troglitazone: the lesson that nobody learned. Diabetologia 49:1-6

34. http://www.britishmuseum.org/explore/online_tours/museum and_exhibition/audio_description_tour/cradle_to_grave by_pharmacopoe.aspx, accessed 29 July 2009

35. Illich I (1976) Limits to medicine. Medical nemesis: the expropriation of health. Marion Boyars, London

36. Todd A, La Cecla F (2002) Ivan Illich: obituary. The Guardian, Monday 9 December

37. Woloshin S, Schwartz LM, Welch HG (2008) Know your chances. Understanding health statistics. University of California Press, Berkeley 